\title{
Electronic Structure of Dendrimer-Au Hybrid Nanoparticle : Hard X-ray Photoemission Study
}

\author{
Yoshiaki Murase ${ }^{1}$, Takanobu Kitagawa ${ }^{1}$, Masaki Imamura ${ }^{1}$, Akinori Tanaka ${ }^{1}$, \\ Hidehiro Yasuda ${ }^{1}$, Yuichi Negishi ${ }^{2}$, Tatsuya Tsukuda ${ }^{3}$, Shigenori Ueda ${ }^{4}$, \\ Yoshiyuki Yamashita ${ }^{4}$, Hideki Yoshikawa ${ }^{4}$, and Keisuke Kobayashi ${ }^{4}$ \\ ${ }^{1}$ Department of Mechanical Engineering, Kobe University, Nada-ku, Kobe 657-8501, Japan \\ ${ }^{2}$ Department of Materials Molecular Science, Institute for Molecular Science, Myodaiji, Okazaki 444-8585, Japan \\ ${ }^{3}$ Catalysis Research Center, Hokkaido University, Kita-ku, Sapporo 001-0021, Japan \\ ${ }^{4}$ National Institute for Materials Science, SPring-8, Sayo, Hyogo 679-5148, Japan \\ Fax: +81-78-803-6123, e-mail: a-tanaka@mech.kobe-u.ac.jp
}

\begin{abstract}
We have carried out the hard X-ray photoemission study of dendrimer-Au hybrid nanoparticles/nanoclusters supported on the highly oriented pyrolytic graphite substrates. From the detailed line-shape analysis for $\mathrm{Au} 4 f$ core-level photoemission spectrum of dendrimer-Au hybrid nanoparticles with mean diameter of $2.6 \mathrm{~nm}$, it is found that $\mathrm{Au} 4 f$ core-level spectrum consists of three components. We attribute these components to the interior Au atoms, surface Au atoms, and surface $\mathrm{Au}$ atoms bonded to dendrimers. Furthermore, we have investigated the valence-band photoemission spectra. From these results, we discuss the electronic structures and interfacial properties of dendrimer-Au hybrid nanoparticles/nanoclusters.

Key words: Hard X-ray photoelectron spectroscopy, Au nanoparticle, Electronic structure
\end{abstract}

\section{INTRODUCTION}

Metal-organic hybrid nanoparticles/nanoclusters are attracted much attention, since they exhibit the distinct optical and electronic properties found in neither bulk nor molecule/atom $[1,2]$. Recently, the synthesis of metallic nanoclusters encapsulated within the dendrimer has been reported by several groups [3-6]. The diameters of these dendrimer-encapsulated metallic nanoclusters are sub-nanometer scale and highly monodispersed, since they are defined by the size of internal cavity of the dendrimer template. Furthermore, these nanoclusters can be easily controlled their solubility, reactivity, and nanocluster-substrate interaction through exchanging the molecule structure and terminal groups on the dendrimer periphery. Therefore, these properties are suitable for the future applications such as drug delivery, biosensing, and catalysis. In order to elucidate their intriguing properties and to develop the future applications, it is necessary to characterize their detailed electronic structures. In this work, we have directly investigated the electronic structures of $\mathrm{Au}$ nanoparticle/nanocluster synthesized using the 2nd generation $\mathrm{OH}$-terminated (G2-OH) poly(amidoamine) (PAMAM) dendrimer as a template. Hard X-ray photoelectron spectroscopy (HX-PES) using high-brilliance synchrotron radiation enables us to carry out the bulk sensitive and high energy-resolution measurements. Moreover, in the hard $\mathrm{x}$-ray region, the photoionization cross-section of Au $6 s p$ electron in the vicinity of Fermi-level is much higher than that of $\mathrm{C} 2 s p$ electron. Therefore, this probe is available to selectively investigate the detailed electronic structures of present Au nanoparticles/nanoclusters. We discuss the detailed electronic structures and the interfacial features of dendrimer-Au hybrid nanoparticles/nanoclusters by means of bulk sensitive high resolution HX-PES.

\section{EXPERIMENT}

Dendrimer-Au hybrid nanoparticles/nanoclusters were prepared using the G2-OH PAMAM dendrimer as a template. Gold chloride (III) was mixed with aqueous solution of G2-OH PAMAM dendrimer with vigorously stirring for 1 day in order to sequester the gold ions into the dendrimer cavities, and then we obtained the preequilibrated gold/dendrimer stock solution. In order to synthesize the relatively larger nanoparticles (method A) [4], $\mathrm{NaBH}_{4}$ (reduction agent) was added to this preequilibrated gold/dendrimer stock solution and solution was stirred at $0{ }^{\circ} \mathrm{C}$ for 3 days. Then, this nanoparticle solution was ultrafilterated with a centrifugation for several times in order to remove the base. In order to synthesize the relatively smaller nanoclusters (method $B$ ) [6], the preequilibrated gold/dendrimer stock solution was incubated at $37^{\circ} \mathrm{C}$ for 3 days to reach growth equilibrium. The size distributions of thus synthesized dendrimer-Au hybrid nanoparticles/nanoclusters were characterized by transmission electron microscopy (TEM) observation at Kobe University and electrospray ionization (ESI) mass spectroscopy at Institute for Molecular Science, Okazaki, Japan. As a further characterization, we performed the optical spectroscopic measurements.

We performed the HX-PES measurements at BL15XU of SPring-8 Facility, Hyogo, Japan. For the HX-PES measurements, the synthesized dendrimer-Au hybrid nanoparticles/nanoclusters were supported on the single-crystalline highly oriented pyrolytic graphite substrates (HOPG) by evaporating the solvent (water) 
(a)
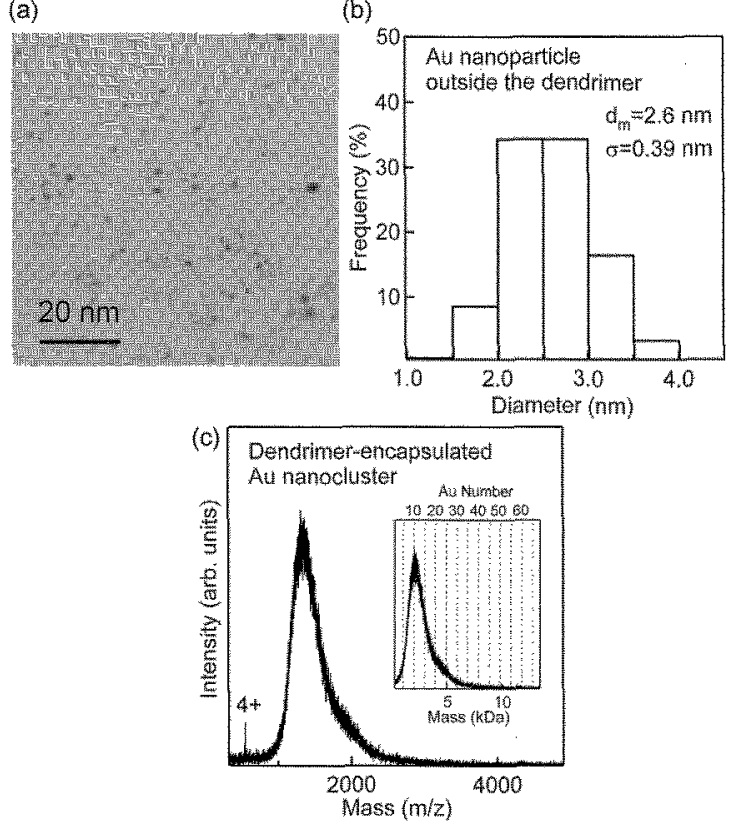

Figure 1. (a) TEM micrograph of dendrimer-Au hybrid nanoparticles. (b) Size distribution of the dendrimer-Au hybrid nanoparticles with mean diameter $d_{\mathrm{m}}$ and standard deviation $\sigma$ of $2.6 \mathrm{~nm}$ and $0.39 \mathrm{~nm}$. (c) ESI mass spectrum of dendrimer-Au hybrid nanocluster and $\mathrm{Au}$ mass number spectrum (in inset).

from the dendrimer-Au hybrid nanoparticles/ nanoclusters solution on the cleaved HOPG surface in a nitrogen-filled glove bag directly connected to the ultrahigh-vacuum photoelectron spectrometer. Then the dendrimer-Au hybrid nanoparticle/nanocluster samples were transferred into the photoemission analysis chamber without exposure to air. The HX-PES measurements were performed with the incident photon energy of $5964.7 \mathrm{eV}$ at room temperature.

\section{RESULTS AND DISCUSSION}

Figure 1 (a) shows the TEM micrograph of dendrimer-Au hybrid nanoparticles synthesized by method A. TEM micrograph shows that present $\mathrm{Au}$ nanoparticle has the spherical shape, and each nanoparticle is separated from neighboring nanoparticles. As shown in Fig. 1 (b), the obtained mean diameter and standard deviation are 2.6 and $0.39 \mathrm{~nm}$, respectively. On the other hand, we could not observe the nanocluster synthesized by method B due to the spatial resolution of the present TEM observation. Therefore, we measured the ESI mass spectrum for the relatively smaller nanoclusters. Figure 1 (c) shows the ESI mass spectrum of $\mathrm{Au}$ nanoclusters synthesized by $\operatorname{method} \mathrm{B}$, as a function of $\mathrm{m} / \mathrm{z}$ (mass divided by charge), and the inset of Fig. 1 (c) shows the Au mass number spectrum as a function of mass. As shown in the inset of Fig. 1 (c), it is found that the mean mass numbers of the present dendrimer-Au hybrid nanoclusters is $\mathrm{Au}_{10}$, and the present sample is accompanied with the finite size distribution. Since the diameter of $\mathrm{Au}_{10}$ nanocluster is smaller than the cavity size of the G2-OH PAMAM

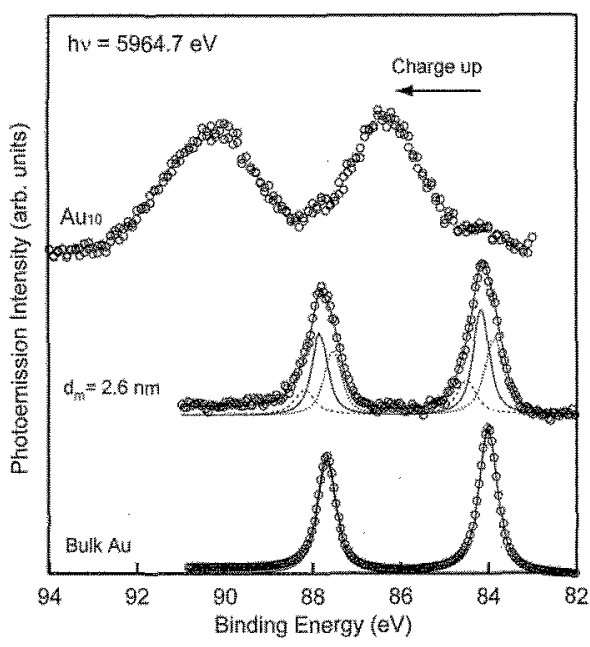

Figure 2. Au $4 f$ core-level hard X-ray photoemission spectra of dendrimer-Au hybrid nanoparticles and dendrimer-encapsulated $\mathrm{Au}$ nanoclusters supported on the HOPG substrate measured with photon energy of $h v=5964.7 \mathrm{eV}$. The bottom spectrum shows the Au $4 f$ core-level spectrum of bulk Au crystalline for a comparison. Photoemission spectrum of bulk $\mathrm{Au}$ is decomposed into only interior component (solid line), and that of $\mathrm{Au}$ nanoparticle with mean diameter of 2.6 $\mathrm{nm}$ is decomposed into interior component (solid line), surface component (dotted line), and interface component (dashed line). The spectrum of $\mathrm{Au}$ nanocluster with mean mass number of $\mathrm{Au}_{10}$ was affected by charge up effect.

dendrimer, $\mathrm{Au}_{10}$ nanocluster is considered to be encapsulated into the dendrimer. In addition, the extinction spectrum of the dendrimer-Au hybrid nanoparticle with mean diameter of $2.6 \mathrm{~nm}$ shows the Mie plasmon resonance, indicative of their metallic electronic structure. On the other hand, the extinction spectrum of the dendrimer-encapsulated $\mathrm{Au}$ nanocluster with mean mass number of $\mathrm{Au}_{10}$ shows no Mie plasmon resonance, indicative of non-metallic electronic structure due to the quantum confinement effect.

Figure 2 shows the Au $4 f$ core-level photoemission spectra of dendrimer-Au hybrid nanoparticles $\left(d_{m}=2.6\right.$ $\mathrm{nm})$ and nanoclusters $\left(\mathrm{Au}_{10}\right)$ supported on the HOPG substrate. Au $4 f$ core-level photoemission spectrum observed for bulk Au polycrystalline is also shown for a comparison. Au $4 f$ core-level spectrum of dendrimer-Au hybrid nanoparticles with mean diameter of $2.6 \mathrm{~nm}$ shows similar spectral feature with that of bulk $\mathrm{Au}$ crystalline, however the spectrum width is wider than that of bulk $\mathrm{Au}$ crystalline. In order to discuss the detailed spectral features, we have carried out the line-shape analyses of $\mathrm{Au} 4 f$ core-level photoemission spectra by a least square method. The detailed procedure of these line-shape analyses for Au $4 f$ core-level spectra has been described elsewhere $[7,8]$. As shown in Fig. 2, it is found that the spectrum of the dendrimer-Au hybrid nanoparticles with mean diameter of $2.6 \mathrm{~nm}$ is reproduced by the three components. The second highest binding-energy component (solid line) is assigned to 
interior Au component, since the spectral feature and binding energy are similar to those of interior component in the Au $4 f$ core-level spectrum observed for bulk $\mathrm{Au}$ crystalline. The lowest binding-energy component (dotted line) is assigned to surface $\mathrm{Au}$ component, since the spectral feature and binding energy are also similar to those of the surface component in the previous XPS spectra for bulk $\mathrm{Au}[8]$. However, the contribution from this surface component to HX-PES spectrum of bulk $\mathrm{Au}$ is negligible due to their bulk sensitivity. On the other hand, from the analogy of previous alkanethiolate-passivated $\mathrm{Au}$ nanoparticles [8], it is considered that the highest binding-energy component (dashed line) is attributed to surface $\mathrm{Au}$ atoms bonded to dendrimer (interface component). This interface component is accompanied with a chemical shift to higher binding-energy side relative to interior component. This means that the chemical states in the interfacial $\mathrm{Au}$ atoms terminated with the dendrimer is different with the interior $\mathrm{Au}$ atoms, and that a chemical reaction (chemisorption) exists between $\mathrm{Au}$ nanoparticles and the hydroxyl terminal groups on the dendrimer periphery. That is, the charge-transfer occurs from $\mathrm{Au}$ nanoparticle to hydroxyl terminal groups on the dendrimer periphery. Since both contributions from surface $\mathrm{Au}$ atoms bonded and non-bonded to dendrimers coexist in the $\mathrm{Au} 4 f$ photoemission spectrum, it is considered that the dendrimers locally bond to the surface $\mathrm{Au}$ atoms of Au nanoparticles. On the other hand, $\mathrm{Au} \quad 4 f$ photoemission spectrum of dendrimer-encapsulated $\mathrm{Au}$ nanocluster with mean mass number of $\mathrm{Au}_{10}$ shifts to higher binding-energy side, and exhibits significantly different spectral features compared to that of bulk Au. It is considered that HX-PES spectrum of dendrimer encapsulated $\mathrm{Au}$ nanoclusters are affected by charge up effect, since the present nanoclusters have the non-metallic electronic structure, and are encapsulated by G2-OH PAMAM dendrimer with the non-electronic conductive property.

Figure 3 (a) shows the HX-PES spectra in the valence-band region of Au nanoparticles stabilized outside the dendrimers and $\mathrm{Au}$ nanoclusters encapsulated into the dendrimers. The photoemission spectrum observed for bulk Au polycrystalline is also shown a comparison in Fig. 3 (a). The spectral features around $2-8 \mathrm{eV}$ binding energy are assigned to $\mathrm{Au}$ $5 d$-derived states. These Au $5 d$-features dominate the valence-band spectra, since their cross-sections are higher than those of $\mathrm{Au} 6 s, \mathrm{C} 2 s$, and $\mathrm{C} 2 p$ electronic states in the relevant photon energy region. As shown in Fig. 3 (a), Au $5 d$-derived features of the bulk $\mathrm{Au}$ crystalline display the well-defined $d$-band doublets. On the other hand, the apparent spin-orbit splitting of $\mathrm{Au}$ nanoparticle with mean diameter of $2.6 \mathrm{~nm}$ is narrower than that of Au crystalline. It has been well established that the noble-metal $d$ - $d$ interaction in bulk crystalline is very strong and $\mathrm{Au} 5 d$ band width and associated apparent spin-orbit splitting decreases with decreasing the coordination number of the nearest atoms [9]. The apparent spin-orbit splitting is described as $\Delta_{5 \mathrm{~d}}=\left(\Delta_{\text {band }}{ }^{2}\right.$ $+\Delta_{\text {s.o. }}{ }^{2}$ ), where $\Delta_{\text {band }}$ is the band broadening contribution and $\Delta_{\text {s.o. }}$ is the true atomic spin-orbit splitting. The band-broadening contribution $\Delta_{\text {band }}$ decreases with the number of nearest neighbors. Since the surface atoms (a)

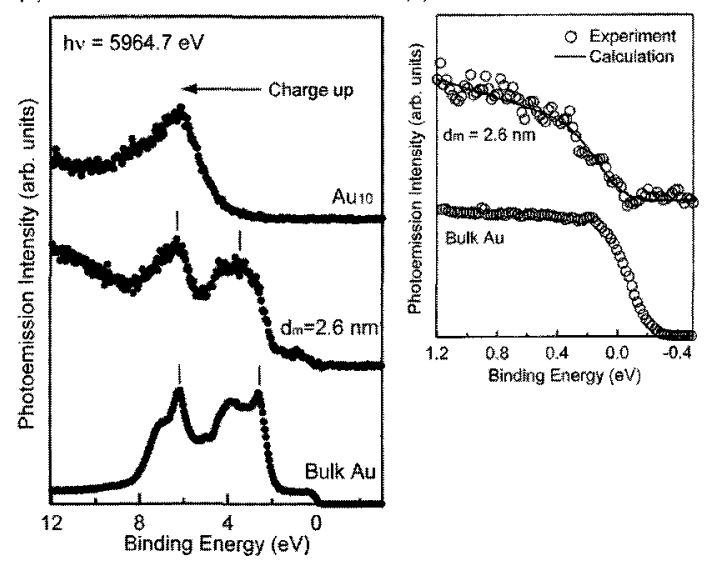

Figure 3. (a) Valence-band hard X-ray photoemission spectra of dendrimer-Au hybrid nanoparticles and dendrimer-encapsulated $\mathrm{Au}$ nanoclusters supported on the HOPG substrate measured with photon energy $h v=$ $5964.7 \mathrm{eV}$. The bottom spectrum shows the valence-band photoemission spectrum of bulk $\mathrm{Au}$ crystalline for a comparison. (b) Comparison of the calculated results based on the dynamic final state effect model (see text) with the experimental photoemission spectrum of dendrimer-Au hybrid nanoparticles with mean diameter of $2.6 \mathrm{~nm}$. Open circles and solid line show the experimental spectrum and the fit to the experimental spectrum, respectively.

have the smaller number of the nearest neighbor than interior atom, and therefore, the surface $d$-derived band and the associated apparent spin-orbit splitting narrows compared with that of bulk crystalline. Since the relative number of surface to interior atoms in nanoparticle increases with decreasing the nanoparticle size, the apparent spin-orbit splitting of dendrimer-Au hybrid nanoparticle with decreasing the nanoparticle size. While HX-PES spectrum of dendrimer-encapsulated $\mathrm{Au}$ nanocluster is affected by charge up effect, the bandwidth of $\mathrm{Au} 5 d$ band narrows. Furthermore, it is found that the HX-PES spectrum of dendrimer-Au hybrid nanocluster with mean mass number of $\mathrm{Au}_{10}$ shows no metallic Fermi edge indicative of non-metallic electronic structure. This result is consistent with that of optical extinction spectrum.

In order to discuss the detailed electronic structure in the vicinity of Fermi level, we plot the photoemission spectra near the Fermi level obtained on an expanded energy scale in Fig. 3 (b). As shown in Fig. 3 (b), the Fermi-level onset in the photoemission spectrum of dendrimer-Au hybrid nanoparticle with mean diameter of $2.6 \mathrm{~nm}$ on the HOPG substrate is not metallic Fermi edge, with the steep slope being away from the Fermi level. Furthermore, it is found that the slope of the leading edge is different from that of the metallic Fermi edge observed for bulk $\mathrm{Au}$. Since the photoemission spectrum in the vicinity of the Fermi level of the HOPG substrate exhibits the featureless shape reflecting the vanishing semi-metallic density of states toward the Fermi level, the observed spectral feature of 
dendrimer-Au hybrid nanoparticles on the HOPG substrate is not caused by the HOPG substrate, but is characteristic of $\mathrm{Au}$ nanoparticles. In general, the photoemission spectrum directly reflects the change in the electronic structure of the samples (initial-state effect) and is also affected by the final-state effect originating from the positively charged photohole created by the photoionization [10-14]. Therefore, first of all, this photoemission spectrum seemed to indicate that the electronic structures of the present dendrimer-Au hybrid nanoparticles change with the nanoparticles size and exhibit the nonmetallic ones in this diameter regime. However, the optical extinction spectrum of the present dendrimer-Au hybrid nanoparticles exhibits the distinctive Mie plasmon resonance around $2.2 \mathrm{eV}$ photon energy, indicative of a collective motion of valence electrons typical for a metallic material. This means that the observed spectral feature in the vicinity of Fermi level is not due to the initial-state effect originating from the change in the electronic structure. Therefore, it is considered that this spectral feature in the vicinity of the Fermi level of the present dendrimer-Au hybrid nanoparticles originates from the final-state effect due to the photohole left behind in the nanoparticles during the photoemission process. In order to theoretically describe this observation, we analyzed the photoemission spectrum in the vicinity of the Fermi level of dendrimer-Au hybrid nanoparticles using a dynamic final-state effect model $[12,13]$ that takes into account the Coulomb interaction between the photoelectron and photohole with a finite lifetime during the photoemission process [8]. This lifetime reflects the coupling strength between $\mathrm{Au}$ nanoparticles and HOPG substrate. Using this model, we have carried out a fit to the experimental spectrum of the dendrimer-Au hybrid nanoparticles by the least squares method. The fitting line to the experimental spectrum is shown by solid line in Fig. 3 (b). As shown in Fig. 3 (b), this fitting line reproduces the experimental spectrum fairly well. From this calculation, the obtained photohole lifetime of the dendrimer-Au hybrid nanoparticles supported on HOPG substrate is shorter than that of dodecanethiolate-Au systems [7]. It indicates that the interaction between $\mathrm{Au}$ nanoparticles and the HOPG substrate is stronger than that of dodecanethiolate- $\mathrm{Au}$ nanoparticle, because the dendrimers locally bond to the Au nanoparticles.

\section{CONCLUSION}

We have synthesized the dendrimer-Au hybrid nanoparticles/nanoclusters using the G2-OH PAMAM dendrimer as a template, and carried out the bulk-sensitive hard X-ray photoemission studies. From the detailed line-shape analyses for Au $4 f$ core-level photoemission spectrum of dendrimer-Au hybrid nanoparticles with mean diameter of $2.6 \mathrm{~nm}$, it is found that $\mathrm{Au} 4 f$ core-level spectrum consists of the three components. We attribute these components to the interior $\mathrm{Au}$ atoms (interior component), surface $\mathrm{Au}$ atoms (surface component), and surface $\mathrm{Au}$ atoms bonded to dendrimers (interface component). Moreover, it is found that the dendrimers locally bond to the surface $\mathrm{Au}$ atoms of $\mathrm{Au}$ nanoparticles. In the valence-band photoemission spectrum of $\mathrm{Au}$ nanoparticle, we have observed the bandwidth narrowing of $\mathrm{Au} 5 d$-derived band compared to that of bulk $\mathrm{Au}$. This feature originates from the lower averaged nearest $\mathrm{Au}$ coordination numbers with decreasing the nanoparticle diameter. Furthermore, Fermi level onset in the photoemission spectrum of $\mathrm{Au}$ nanoparticle on the HOPG substrates is not metallic Fermi edge, with the steep slope being away from the Fermi level. It is found that the photoemission spectrum is well reproduced by the calculation based on the dynamic final-state effect model, and the obtained photohole lifetime is shorter than that of previous alkanethiolate-Au system. On the other hand, HX-PES spectra of dendrimer-encapsulated $\mathrm{Au}$ nanocluster with mean mass number of $\mathrm{Au}_{10}$ are affected by charge-up effect.

\section{ACKNOWLEDGMENTS}

This work was partially supported by Grant-in-Aids from the Japan Society for the Promotion of Science (JSPS) (No. 19510109 and No.19-1410). The synchrotron radiation experiments were performed at SPring-8 with the approval of National Institute for Materials Science (NIMS) as Innovation-Nanotechnology Network Project of the Ministry of Education, Culture, Sports, Science and Technology (MEXT) (Proposal No. 2007A4902 and No.2007B4907).

\section{REFERENCES}

[1] M. J. Hostetler, J. E. Wingate, C. Zhong, J. E. Harris, R. W. Vachet, M. R. Clark, J. D. Londono, S. J. Green, J. J. Stokes, G, D, Wignall, G. L. Glish, M. D. Porter, N. D. Evans, and R. W. Murray, Langmuir 14, 17 (1998)

[2] M. Alvarez, J. T. Khoury, T. G. Shaaff, M. N. Shafigulin, I. Vezmar, and R. L. Whetten, J. Phys. Chem. 101, 3706 (1997)

[3] Y. G. Kim, J. C. G. Martinez, and R. M. Crooks, Langmuir 21, 5485 (2005).

[4] J. Zheng, C. Zhang, and R. M. Dickson, Phys. Rev. Lett. 93, 077402 (2004).

[5] T. Endo, T. Yoshimura, and K. Esumi, Coll. Int. Sci. 269, 364 (2004).

[6] Y. Bao, C. Zhong, D. M. Vu, J. P. Termirov, R. B Dyer, and J. S. Martinez, J. Phys. Chem. C 111, 12194 (2007).

[7] A. Tanaka, Y. Takeda, M. Imamura, and S. Sato, Phys. Rev. B 68, 195415 (2003).

[8] A. Tanaka, Y. Takeda, T. Nagasawa, and K. Takahashi, Solid State Commun. 126, 191 (2003).

[9] A. Bzowski, T. K. Sham, R. E. Watson, and M. Weinert, Phys. Rev. B 51, 9979 (1995).

[10] M. Seidl, K. H. Meiwes - Broer, and M. Brack, $J$ Chem. Phys. 95, 1295 (1991).

[11] S. L. Qiu, X. Pan, M. Strongin, and P. H. Citrin, Phys. Rev. B 36, 1292 (1987).

[12] H. Hovel, B. Grimm, M. Pollmann, and B. Reihl, Phys. Rev. Lett. 81, 4608 (1998)

[13] H. Hovel, B. Grimm, M. Pollmann, and B. Reihl, Eur. Phys. J. D 9, 595 (1999).

[14] M. G. Mason, Phys. Rev. B 27, 748 (1983). 